\title{
Person-Centred Maternity Care in a Poor-Resource Setting: Evidence from a Cross-Sectional Study in Enugu State, Nigeria
}

Daniel Chukwuemeka Ogbuabor ( $\nabla$ ogbuabordc@gmail.com )

Sustainable Impact Resource Agency https://orcid.org/0000-0002-9617-1538

Chikezie Nwankwor

University of Nigeria - Enugu Campus

\section{Research article}

Keywords: Person-centred care, Maternity care, Respectful maternity care, Responsive care, Facility-based childbirth, Nigeria

Posted Date: May 8th, 2020

DOI: https://doi.org/10.21203/rs.3.rs-25409/v1

License: (c) (i) This work is licensed under a Creative Commons Attribution 4.0 International License.

Read Full License 


\section{Abstract}

Background Women are not getting adequate person-centred maternity care (PCMC) in low-income and middle-income countries despite being important in reducing maternal morbidity and mortality. This study assessed perceptions and predictors of PCMC among childbearing women in Enugu State, Southeast Nigeria.

Methods The study was conducted in two health districts in Enugu State using a descriptive, crosssectional survey design. We conveniently selected 450 childbearing women, within 9 weeks post-partum, from 11 health facilities purposively selected based on high maternal and child health attendance. Data was collected from women using an interviewer administered 30-item PCMC scale (scores 0-90) consisting of: dignity and respect (6 items, scores $0-18)$, communication and autonomy (9 items, scores $0-27)$, and supportive care (15 items, scores $0-45)$. We categorized full PCMC and each sub-scale into "low, medium and high" using 25th and 75th percentile of the summative scores. The questionnaire also collected data on women characteristics, facility characteristics and service type. Data were analysed using descriptive statistics, $\mathrm{t}$ - tests, analysis of variance and Generalized Linear Models.

Results Women had medium scores on full PCMC scale and sub-scales. The lowest score was in communication and autonomy. A quarter of women perceived PCMC as high. Marrying at age 2029 years $(\beta=5.83, \rho=0.002)$, self-employed women $(\beta=-10.23, \rho=0.004)$, starting antenatal care in the third trimester $(\beta=-7.93, \rho=0.008)$, high participation in household decisions $(\beta=-4.98, \rho=0.011)$, domestic violence experience $(\beta=5.41, \rho=0.002)$, delivery at health centre $(\beta=7.09, \rho=0.000)$, delivery at private/mission hospital $(\beta=17.47, \rho=0.000)$, delivery by non-skilled attendant $(\beta=-9.61, \rho=0.001)$; delivery by community health workers $(\beta=-7.65, \rho=0.001)$, and experience of pregnancy complication ( $\beta$ $=6.20, \rho=0.028)$ predicted PCMC.

Conclusions Person-centred maternity care was generally inadequate. Improving PCMC would entail sustaining dignified care, enhancing provider-patient communication, addressing facility-level drivers, increasing women's personal empowerment and self-employment, timely initiation of antenatal care, and paying attention to women with pregnancy complication.

\section{Background}

Person-centred maternity care (PCMC), defined as "maternity care that is respectful of and responsive to individual women and their families' preferences, needs, and values" [1], is an effective strategy for improving quality of care experienced by women during childbirth in health facilities in low- and middleincome countries [2-5]. PCMC comprises three domains of patient experience: autonomy and communication, respect and dignity, and supportive care [1, 6]. Its main objectives are to reduce mistreatment, abuse, disrespect and neglect of women during labour and childbirth in health facilities; improve the use of skilled birth attendants and promote positive childbirth experiences [6, 7]. Improvements in the quality of maternity care and access to skilled birth attendance contribute to a 
reduction in maternal mortality ratio and attainment of target three of Sustainable Development Goals [3, 7]. However, neglect of PCMC leads to disparities in use of skilled birth attendance and maternal and neonatal outcomes $[2,8,9]$.

Although several qualitative studies have described disrespectful, abusive, and neglectful treatment of women during facility-based childbirth, few quantitative studies measuring PCMC indicate that women are not getting adequate PCMC in low-income and middle-income countries $[1,6,10,11]$. Overall, $11-40 \%$ of women were treated with respect few times or never; $14-39 \%$ received friendly treatment few times or never; $11-18 \%$ were verbally abused at least once; $2-4 \%$ reported physically abuse; $11-47 \%$ did not receive adequate visual privacy; and $15-38 \%$ felt their health records were kept confidential few times or never $[6,10,11]$. Other studies found 19-98\% prevalence of disrespect and abuse among women during childbirth in health facilities [12-19]. About $87-98 \%$ of women reported limited introduction of self by providers; $47-69 \%$ reported providers did not often call them by names; $17-77 \%$ were poorly involved in their care; $50-87 \%$ received unconsented procedures; $29-82 \%$ received poor explanation of medicines; and $36-74 \%$ felt unable to ask providers questions $[6,10,11]$. Also, $13-26 \%$ of women reported long time to access care; $13-51 \%$ lack of labour support; $13-78 \%$ limited childbirth support; $33-54 \%$ poor control of pain; 36-79\% limited emotional support; $18-61 \%$ inadequate workforce; and $13-94 \%$ dirty health facilities $[6,10,11]$. Mean full PCMC scores ranged from low in Ghana to moderately high in Kenya and India $[1,6,10,11]$. Perception of dignity and respect was high; communication and autonomy, low to moderately high; and supportive care, moderately high to high $[1,6,10,11]$.

Broadly, three context-specific factors, which shape patients' needs, expectation and values, affect person-centred maternity care namely facility characteristics, service type, and patient characteristics [5]. Patient characteristics include socio-demographic characteristics, clinical history and prior health careseeking behaviour [5]. Age and education explain differences in patient's experiences of care, perception of quality of care and satisfaction with care [20]. Women with high socio-economic status are usually personally empowered; live in areas with high quality of care; tend to have relationships with healthcare providers, narrow social power between women and providers, higher expectation of care, and capacity to advocate for high quality care $[8,21]$. Higher incidents of disrespectful and abusive care were reported from women from lower socio-economic strata in Ethiopia and Pakistan [18, 22, 23]. Women who were younger and less educated were most at risk of disrespect and abuse during childbirth in Ghana, Guinea, Myanmar, and Nigeria [17]. Perception of PCMC varied with socio-economic status and education in Kenya, India and Ghana $[8,11]$. Furthermore, gainful employment, being married and absence of domestic violence experience predicted higher PCMC among childbearing women [8]. In this study, we hypothesize that socio-economic factors, experience of domestic violence and participation in household decisions will be associated with PCMC.

Facility characteristics such as type of facilities, types of providers, number of patients seen, providerpatient ratio, availability of services and resources may modify women's experiences of maternity care [5, 24]. Whereas indices of clinical quality of maternal health were higher in hospitals than health centres [25-27], interpersonal quality of maternal healthcare was higher in private than public hospitals $[25,28]$. 
Women who delivered in health centres reported higher PCMC than those who were delivered in public hospitals in Kenya and India, but no significant difference was observed in Ghana $[8,11]$. Whereas women who delivered in private health facilities reported higher PCMC than those who were delivered in public hospitals in Kenya [8, 11], no significant difference was observed in Ghana [11]. Disrespect and abuse were more likely to be reported by women delivering at hospitals than health centres in Ethiopia [18]. In Pakistan, the risk of reporting disrespect and abuse was twice in public health facilities as compared to private [23]. Mothers who gave birth in primary level facilities have a tendency to be more satisfied than those who gave birth in hospitals [29]. In this study, we hypothesize that PCMC will be lower in public hospitals than in health centres and private hospitals.

Evidence that type of services such as non-emergency or emergency care and experience of pregnancy complications influence PCMC are mixed. In Pakistan, the type of delivery service did not influence women's experiences of disrespect and abuse [23]. In contrast, women who experienced pregnancy complications reported higher PCMC than those who did not experience complications in Ghana, but not in Kenya and India [11]. However, severe pregnancy complication significantly predicted higher PCMC [8]. Also, In Gambia, women who had a normal vaginal delivery were more likely to have a higher perception of autonomy and supportive care than those who had instrumental delivery [30]. Conversely, higher incidents of disrespectful and abusive care were reported from women who had and who had a delivery with complications, longer labour durations and Caesarean birth [19, 22, 31, 32]. Our proposition in this study is that women with pregnancy complications will have significantly lower PCMC than those without complications.

In Nigeria, increase in skilled birth attendance has not been matched with enough quality of maternity care. A systematic review of studies on respectful maternity care in Nigeria indicate that mistreatment of women during childbirth are common and not only undermine utilization of health facilities for delivery but also create psychological distance between women and health providers [16]. Yet, disrespect and abuse of women at childbirth has not been comprehensively studied in the Nigerian health system [12, 16]. Equally, the broad concept of PCMC is still nascent in Nigeria, and to our knowledge, there is no study that has investigated the perception and predictors of PCMC in Nigeria. There is a need for evidence that extends studies on respectful maternity care to include effective communication and autonomy, and supportive care during labour and childbirth using a validated PCMC scale $[1,6,7]$. This study, therefore, assessed perceptions and predictors of PCMC among childbearing women in Enugu State, South-east Nigeria. As patient experience is sensitive to differences in quality care across different providers, institutions and time (5), evidence from this study, on what works for PCMC and what does not, will be helpful to health decision-makers, providers and service users in identifying gaps, designing interventions to promote positive childbirth experiences and evaluating changes in quality of maternity care.

\section{Methods}

\section{Study setting}


The study took place in two districts of Enugu State, South-east Nigeria. We purposively chose two health districts: Enugu metropolis, the capital city, and Isi-Uzo district following our previous maternal and child healthcare study [33], and to compare urban with rural settings. The two districts have, each a general hospital and a network of cottage hospitals and primary health facilities. In 2019, the estimated population of Enugu State was about 4.8 million people. Enugu Metropolis and Isi-Uzo had 1,061,256 and 217,952 populations respectively, out of which women of childbearing age constitute $47.2 \%$ and $43.1 \%$ respectively [34]. About $94.7 \%$ of women in Enugu received ante natal care from a skilled provider, while skill birth attendance is about $93 \%$ [34]. However, the maternal mortality rate in Enugu is 645/100, 000 live births [35], higher than the national rate of 512/100, 000 live births [34].

\section{Research design}

The study adopted a facility-based cross-sectional survey design using an interviewer administered questionnaire.

\section{Study population and sampling strategy}

Women of childbearing age (WCBA), aged 15-49 years, who delivered in 9 weeks preceding the study constituted the study population. Using $54.5 \%$ prevalence for non-consented care among women during childbirth from a previous study in Enugu [12], 95\% confidence limit, allowable error of 0.05 and 10\% nonresponse rate, the minimum sample size was calculated to be 419 . We, however, sampled 450 eligible WCBA.

In each district, we purposively selected a general hospital and four primary health centres with the highest maternal and child healthcare attendance based on routine health management information system. Additionally, the sample in Enugu metropolis purposively included the state teaching hospital because of its central location which made it very accessible. The sample was equally allocated to the two districts. Eligible WCBA were recruited by convenience at the health facilities as they leave immunisation clinics using healthcare providers as gatekeepers.

\section{Data collection.}

Data was collected from eligible WCBA from January to March 2019 using an interviewer administered PCMC scale made up of 30 items measuring three domains of PCMC: dignity and respect (6 items), communication and autonomy (9 items), and supportive care (15 items). The PCMC scale has been validated in similar low-resource context $(1,6)$. In our sample, Kaiser-Meyer-Olkin measure of sampling adequacy was $0.963(X 2=16083.11, \rho=0.000)$. On exploratory factor analysis with principal component analysis, all 30 items yielded communalities $\geq 0.4$, which was deemed adequate [36]. The cumulative variance was $73.3 \%$. Oblimin rotation with Kaiser Normalization showed all 30 items loaded $\geq 0.4$ and were retained since a rotated factor loading of 0.32 is considered significant [36]. The reliability coefficient of the PCMC scale was 0.964. Each item is on a 4-point response scale-0: "no, never," 1: "yes, a few times," 2: "yes, most of the time," and 3: "yes, all the time." For each respondent, responses from the 
PCMC scale were summed up into one composite PCMC score with interval-like properties [37]. The possible score on the PCMC scale range from 0 to 90 , with a lower score implying poorer PCMC. The range of possible scores on the sub-PCMC scales are: 0-18, 0-27 and 0-45 for respect and dignity, communication and autonomy, and supportive care correspondingly. We categorized full PCMC and each sub-scale into "low, medium and high". Low was defined as scores in the approximate lower 25th percentile and scores in the top 75th percentile defined as high [38].

The questionnaire also included information on socio-demographic characteristics such as age, marital status, residence, religion, age at marriage, education, literacy, occupation, partner's education, partner's occupation, and maternal health care-seeking behaviour. Other information collected include facility characteristics (facility type and provider type), service types, household wealth index, women's participation in household decisions, domestic violence tolerance and experience.

Household wealth index was measured using 11 questions on Nigeria equity tool and its accompanying syntax used to create wealth quintiles [39]. Participation in household decision-making was assessed using questions on different household decisions such as respondent's healthcare, visit to family and relatives, large household purchases, how respondent's earning will be used, and how husband/partner's earning will be used [40]. Each question was assigned the following scores: 0 - if the decision was made by husband/partner alone, someone else or other; 1 - if the decision was jointly made by respondent and husband/partner; and 2 - if the respondent alone made the decision. Participation score ranged from 010. Also, attitudes towards domestic violence were measured using five variables describing whether beating was justified if the wife: goes out without telling her husband; neglects the children; argues with her husband; refuses sex with her husband; and burns food [40]. Women who answered 'Yes' and 'Don't know' were scored 0 while women who responded 'No' were scored 1. Domestic violence tolerance score ranged from $0-5$. The value of either the participation score or domestic violence tolerance was transformed into 0-1 interval [40]. The median values were used to dichotomise the scores into low and high participation as well as domestic violence tolerant and intolerant categories. The questionnaire was pre-tested on women with recent deliveries in a different district to check for ease of understanding. Five trained research assistants administered the questionnaires, while the authors checked questionnaires for completeness daily.

\section{Data analysis.}

Data were analyzed using SPSS (version 20, IBM, New York, USA). Characteristics of respondents were presented using frequencies and percentages. Mean PCMC scores and standard deviation were calculated and compared across various socio-demographic characteristics of respondents, facility characteristics and service type using t- tests and analysis of variance (ANOVA). Parametric tests were deemed appropriate since the single composite PCMC scores have interval-like properties. Generalized Linear Models was used to the test relationship between PCMC and the parameters that were significant on bivariate analysis. Statistical significance was set at alpha 0.05 level.

\section{Ethical consideration}


The study was approved by the Health Research Ethics Committee of University of Nigeria Teaching Hospital, Enugu, Nigeria. Written, informed consent was obtained from all respondents.

\section{Results}

\section{Characteristics of respondent}

The response rate was $100 \%$. Table 1 shows the characteristics of respondents. About $58 \%$ of women were in the 20-29 years age group and educated to at least secondary or vocational school. Most women were married, Christians, Igbo, and married in their $20 \mathrm{~s}$. Nearly half of women could read and write very well. About a fifth of women were unemployed. Over $50 \%$ of women had low participation in household decisions. Whereas $90 \%$ of women were intolerant to domestic violence, about $30 \%$ had experienced domestic violence. Over $50 \%$ of women started antenatal care during or after the second trimester. Few women $(10.2 \%)$ were delivered by non-skilled provider, while about $8 \%$ reported pregnancy complications. 
Table 1

Characteristics of respondents

\begin{tabular}{|c|c|c|c|}
\hline \multicolumn{2}{|l|}{ Parameters } & \multirow{2}{*}{$\begin{array}{l}\text { Frequency (n) } \\
25\end{array}$} & \multirow{2}{*}{$\begin{array}{l}\text { Percent (\%) } \\
5.6\end{array}$} \\
\hline Age & $15-19$ years & & \\
\hline & $20-29$ years & 259 & 57.6 \\
\hline & $30-49$ years & 166 & 36.9 \\
\hline \multirow[t]{2}{*}{ Marital status } & Married & 444 & 98.7 \\
\hline & Not married & 6 & 1.3 \\
\hline \multirow[t]{2}{*}{ Religion } & Christianity & 447 & 99.3 \\
\hline & Muslim & 3 & 0.7 \\
\hline \multirow[t]{2}{*}{ Ethnicity } & Igbo & 444 & 98.7 \\
\hline & Others & 6 & 1.3 \\
\hline \multirow[t]{2}{*}{ Residence } & Urban & 225 & 50.0 \\
\hline & Rural & 225 & 50.0 \\
\hline \multirow[t]{3}{*}{ Age at marriage } & $15-19$ years & 93 & 20.7 \\
\hline & $20-29$ years & 322 & 71.5 \\
\hline & $30-49$ years & 35 & 7.8 \\
\hline \multirow[t]{4}{*}{ Parity } & 1 & 134 & 29.8 \\
\hline & 2 & 130 & 28.9 \\
\hline & 3 & 103 & 22.9 \\
\hline & $\geq 4$ & 83 & 18.4 \\
\hline \multicolumn{4}{|c|}{ Educational level of respondent } \\
\hline & No school/ primary & 55 & 12.2 \\
\hline & Secondary/Vocational & 262 & 58.2 \\
\hline & Tertiary & 133 & 29.6 \\
\hline \multirow[t]{3}{*}{ Literacy rate writing } & No, cannot write & 59 & 13.1 \\
\hline & Yes, with some difficulty & 157 & 34.9 \\
\hline & Yes, very well & 234 & 52.0 \\
\hline \multirow[t]{2}{*}{ Literacy rate reading } & No, cannot read & 58 & 12.9 \\
\hline & Yes, with some difficulty & 155 & 34.4 \\
\hline
\end{tabular}




\begin{tabular}{|c|c|c|c|}
\hline \multicolumn{2}{|l|}{ Parameters } & \multirow{2}{*}{$\begin{array}{l}\text { Frequency (n) } \\
237\end{array}$} & \multirow{2}{*}{$\begin{array}{l}\text { Percent (\%) } \\
52.7\end{array}$} \\
\hline & Yes, very well & & \\
\hline \multirow[t]{5}{*}{ Wealth index } & Poorest & 66 & 14.7 \\
\hline & Poorer & 59 & 13.1 \\
\hline & Middle & 57 & 12.7 \\
\hline & Richer & 68 & 15.1 \\
\hline & Richest & 200 & 44.4 \\
\hline \multirow[t]{5}{*}{ Occupation of respondent } & Unemployed/student & 89 & 19.8 \\
\hline & Self employed & 148 & 32.9 \\
\hline & Salaried worker & 56 & 12.4 \\
\hline & Artisan/petty trading & 97 & 21.6 \\
\hline & Farmer & 60 & 13.3 \\
\hline \multirow[t]{2}{*}{ Employment of respondent } & Unemployed & 89 & 19.8 \\
\hline & Employed & 361 & 80.2 \\
\hline \multirow[t]{5}{*}{ Partner's occupation } & Unemployed/student & 17 & 3.8 \\
\hline & Self employed & 189 & 42.0 \\
\hline & Salaried worker & 96 & 21.3 \\
\hline & Artisan/petty trading & 99 & 22.0 \\
\hline & Farmer & 49 & 10.9 \\
\hline \multicolumn{4}{|l|}{ Partner's educational level } \\
\hline & No school/ primary & 84 & 18.7 \\
\hline & Secondary/Vocational & 226 & 50.2 \\
\hline & Tertiary & 140 & 31.1 \\
\hline \multirow[t]{2}{*}{ Health insurance } & No & 412 & 91.6 \\
\hline & Yes & 38 & 8.4 \\
\hline \multirow[t]{2}{*}{ Participation in decision making } & Low participation & 258 & 57.3 \\
\hline & High participation & 192 & 42.7 \\
\hline \multirow[t]{2}{*}{ Domestic violence attitude } & DV tolerance & 45 & 10.0 \\
\hline & DV intolerance & 405 & 90.0 \\
\hline
\end{tabular}




\begin{tabular}{|c|c|c|c|}
\hline \multicolumn{2}{|l|}{ Parameters } & \multirow{2}{*}{$\begin{array}{l}\text { Frequency (n) } \\
316\end{array}$} & \multirow{2}{*}{$\begin{array}{l}\text { Percent (\%) } \\
70.2\end{array}$} \\
\hline Domestic violence experience & No & & \\
\hline & Yes & 134 & 29.8 \\
\hline \multirow[t]{3}{*}{ Delivery facility Type } & Public hospital & 160 & 35.6 \\
\hline & Public health centre & 130 & 28.9 \\
\hline & Private/mission & 160 & 35.6 \\
\hline \multirow[t]{4}{*}{ Provider type } & Nurse/midwife & 117 & 26.0 \\
\hline & Doctor & 86 & 19.1 \\
\hline & Community health worker & 201 & 44.7 \\
\hline & Non-skilled attendant & 46 & 10.2 \\
\hline \multirow[t]{2}{*}{ Pregnancy complication } & No & 413 & 91.8 \\
\hline & Yes & 37 & 8.2 \\
\hline \multirow[t]{2}{*}{ Previous facility delivery } & No & 172 & 38.2 \\
\hline & Yes & 278 & 61.8 \\
\hline \multirow[t]{4}{*}{ Trimester of first ANC } & No ANC & 5 & 1.1 \\
\hline & First trimester & 200 & 44.4 \\
\hline & Second trimester & 213 & 47.3 \\
\hline & Third trimester & 32 & 7.1 \\
\hline \multirow[t]{4}{*}{ Number of ANC visits } & No ANC & 8 & 1.8 \\
\hline & $<4$ & 37 & 8.2 \\
\hline & 4 or 5 & 161 & 35.8 \\
\hline & $\geq 6$ & 244 & 54.2 \\
\hline \multirow[t]{5}{*}{ Postpartum length } & 1 week & 43 & 9.6 \\
\hline & 2 weeks & 12 & 2.7 \\
\hline & 3 weeks & 5 & 1.1 \\
\hline & 4 weeks & 25 & 5.6 \\
\hline & 5-9 weeks & 365 & 81.1 \\
\hline
\end{tabular}




\section{Distribution of individual PCMC items among women $(\mathrm{N}=$ 450)}

As shown in Table 2, most women were treated with respect, but $20 \%$ were verbally abused, and seven percent were physically abused. Most women reported good visual privacy and record confidentiality. 
Table 2

Distribution of individual PCMC items among women

\begin{tabular}{|c|c|c|c|c|}
\hline PCMC domain & Item & Response & $\begin{array}{l}\text { Frequency } \\
\text { (n) }\end{array}$ & $\begin{array}{l}\text { Percent } \\
\text { (\%) }\end{array}$ \\
\hline \multirow[t]{12}{*}{ Dignity and respect } & \multirow[t]{2}{*}{ Treated with respect } & $\begin{array}{l}\text { Few times or } \\
\text { never }\end{array}$ & 94 & 20.9 \\
\hline & & $\begin{array}{l}\text { Most or all the } \\
\text { time }\end{array}$ & 356 & 79.1 \\
\hline & \multirow[t]{2}{*}{ Friendly } & $\begin{array}{l}\text { Few times or } \\
\text { never }\end{array}$ & 30 & 6.7 \\
\hline & & $\begin{array}{l}\text { Most or all the } \\
\text { time }\end{array}$ & 420 & 93.3 \\
\hline & \multirow[t]{2}{*}{ Verbal abuse } & Never & 359 & 79.8 \\
\hline & & At least once & 91 & 20.2 \\
\hline & \multirow[t]{2}{*}{ Physical abuse } & Never & 418 & 92.9 \\
\hline & & At least once & 32 & 7.1 \\
\hline & \multirow[t]{2}{*}{ Visual privacy } & $\begin{array}{l}\text { Few times or } \\
\text { never }\end{array}$ & 100 & 22.2 \\
\hline & & $\begin{array}{l}\text { Most or all the } \\
\text { time }\end{array}$ & 350 & 77.8 \\
\hline & \multirow[t]{2}{*}{ Record confidentiality } & $\begin{array}{l}\text { Few times or } \\
\text { never }\end{array}$ & 100 & 22.2 \\
\hline & & $\begin{array}{l}\text { Most or all the } \\
\text { time }\end{array}$ & 350 & 77.8 \\
\hline \multirow[t]{7}{*}{$\begin{array}{l}\text { Autonomy and } \\
\text { communication }\end{array}$} & \multirow[t]{2}{*}{ Introduce self } & $\begin{array}{l}\text { Few times or } \\
\text { never }\end{array}$ & 170 & 37.8 \\
\hline & & $\begin{array}{l}\text { Most or all the } \\
\text { time }\end{array}$ & 280 & 62.2 \\
\hline & \multirow[t]{2}{*}{ Called by name } & $\begin{array}{l}\text { Few times or } \\
\text { never }\end{array}$ & 146 & 32.4 \\
\hline & & $\begin{array}{l}\text { Most or all the } \\
\text { time }\end{array}$ & 304 & 67.6 \\
\hline & \multirow[t]{2}{*}{ Involvement in care } & $\begin{array}{l}\text { Few times or } \\
\text { never }\end{array}$ & 142 & 31.6 \\
\hline & & $\begin{array}{l}\text { Most or all the } \\
\text { time }\end{array}$ & 308 & 68.4 \\
\hline & $\begin{array}{l}\text { Consent to } \\
\text { procedures }\end{array}$ & $\begin{array}{l}\text { Few times or } \\
\text { never }\end{array}$ & 110 & 24.4 \\
\hline
\end{tabular}




\begin{tabular}{|c|c|c|c|c|}
\hline PCMC domain & Item & Response & $\begin{array}{l}\text { Frequency } \\
\text { (n) }\end{array}$ & $\begin{array}{l}\text { Percent } \\
\text { (\%) }\end{array}$ \\
\hline & & $\begin{array}{l}\text { Most or all the } \\
\text { time }\end{array}$ & 340 & 75.6 \\
\hline & \multirow[t]{2}{*}{ Delivery position } & $\begin{array}{l}\text { Few times or } \\
\text { never }\end{array}$ & 392 & 87.1 \\
\hline & & $\begin{array}{l}\text { Most or all the } \\
\text { time }\end{array}$ & 58 & 12.9 \\
\hline & \multirow[t]{2}{*}{ Language } & $\begin{array}{l}\text { Few times or } \\
\text { never }\end{array}$ & 93 & 20.7 \\
\hline & & $\begin{array}{l}\text { Most or all the } \\
\text { time }\end{array}$ & 357 & 79.3 \\
\hline & \multirow[t]{2}{*}{$\begin{array}{l}\text { Explain } \\
\text { exams/procedure }\end{array}$} & $\begin{array}{l}\text { Few times or } \\
\text { never }\end{array}$ & 111 & 24.7 \\
\hline & & $\begin{array}{l}\text { Most or all the } \\
\text { time }\end{array}$ & 339 & 75.3 \\
\hline & \multirow[t]{2}{*}{ Explain medicine } & $\begin{array}{l}\text { Few times or } \\
\text { never }\end{array}$ & 111 & 24.7 \\
\hline & & $\begin{array}{l}\text { Most or all the } \\
\text { time }\end{array}$ & 339 & 75.3 \\
\hline & \multirow[t]{2}{*}{ Able to ask question } & $\begin{array}{l}\text { Few times or } \\
\text { never }\end{array}$ & 95 & 21.1 \\
\hline & & $\begin{array}{l}\text { Most or all the } \\
\text { time }\end{array}$ & 355 & 78.9 \\
\hline \multirow[t]{7}{*}{ Supportive care } & \multirow[t]{2}{*}{ Time to care } & $\begin{array}{l}\text { Few times or } \\
\text { never }\end{array}$ & 348 & 77.3 \\
\hline & & $\begin{array}{l}\text { Most or all the } \\
\text { time }\end{array}$ & 102 & 22.7 \\
\hline & \multirow[t]{2}{*}{ Labour support } & $\begin{array}{l}\text { Few times or } \\
\text { never }\end{array}$ & 335 & 74.4 \\
\hline & & $\begin{array}{l}\text { Most or all the } \\
\text { time }\end{array}$ & 115 & 25.6 \\
\hline & \multirow[t]{2}{*}{ Childbirth support } & $\begin{array}{l}\text { Few times or } \\
\text { never }\end{array}$ & 373 & 82.9 \\
\hline & & $\begin{array}{l}\text { Most or all the } \\
\text { time }\end{array}$ & 77 & 17.1 \\
\hline & Talk about feeling & $\begin{array}{l}\text { Few times or } \\
\text { never }\end{array}$ & 111 & 24.7 \\
\hline
\end{tabular}


Item

Response

Frequency

(n)

339

Most or all the time

Support anxiety

Few times or never

Most or all the time

Attention when need
help

Few times or
never

Most or all the time

Took best care

Control pain

Trust

Enough staff

Crowded

Cleantines

Cleanliness

Clean water

Few times or
never

191

42.4

259

57.6

90

20.0

360

80.0

90

20.0

Most or all the

360

80.0

time

Few times or
never

205

45.6

Most or all the
time

245

54.4

Few times or never

88

19.6

Most or all the

362

time

Few times or never

131

29.1

Most or all the time

Few times or never

Most or all the 193 time

Clean water

Few times or
never

Most or all the time

Few times or never

75.3

2.4

0.0

Percent 


\begin{tabular}{|llllc|}
\hline PCMC domain & Item & Response & \multicolumn{1}{c|}{$\begin{array}{l}\text { Frequency } \\
\text { (n) }\end{array}$} & $\begin{array}{l}\text { Percent } \\
\text { (\%) }\end{array}$ \\
\hline Electricity & $\begin{array}{l}\text { Most or all the } \\
\text { time }\end{array}$ & 145 & 32.2 \\
\hline Safe & $\begin{array}{l}\text { Few times or } \\
\text { never }\end{array}$ & 104 & 23.1 \\
\hline $\begin{array}{l}\text { Most or all the } \\
\text { time }\end{array}$ & 346 & 76.9 \\
\hline & $\begin{array}{l}\text { Few times or } \\
\text { never }\end{array}$ & 90 & 20.0 \\
\hline $\begin{array}{l}\text { Most or all the } \\
\text { time }\end{array}$ & 360 & 80.0 \\
\hline
\end{tabular}

Most women reported that providers introduced themselves, called women by their names, involved women in care decisions, sought consent to procedures, talked to women in language that women understood, explained examinations and medicines (Table 2). However, whereas $79 \%$ were able to ask questions, only $13 \%$ of women were able to choose their delivery position.

Most women indicated that providers promptly initiated care, paid attention when needed, talked to women about their feeling, took the best care of them and could be trusted (Table 2). Most women also reported that health facilities were safe, clean, had electricity and clean water. Although most women reported that health facilities have few staff, health facilities did not allow labour and childbirth support (Table 2).

\section{Distribution of full PCMC scale and sub-scales}

Table 3 shows that about a quarter of women perceived PCMC as high. Nearly two-third of women perceived respect and dignity as high. While less than a third of women perceived communication and autonomy as high, almost a third perceived supportive care as high. As shown in Table 4, women had medium scores on full PCMC scale and sub-scales. 
Table 3

Total PCMC and sub-scale cores

\begin{tabular}{|lll|}
\hline Outcome & Frequency (n) & Percent $^{\mathrm{a}}$ (\%) \\
\hline Total PCMC score & $\mathrm{N}=450$ & \\
\hline Low & 109 & 24.2 \\
\hline Medium & 228 & 50.7 \\
\hline High & 113 & 25.1 \\
\hline Dignity and respect sub-scale score & $\mathrm{N}=450$ & \\
\hline Low & 107 & 23.8 \\
\hline Medium & 48 & 10.7 \\
\hline High & 295 & 65.6 \\
\hline Communication and autonomy sub-scale score & $\mathrm{N}=450$ & \\
\hline Low & 106 & 23.6 \\
\hline Medium & 210 & 46.7 \\
\hline High & 134 & 29.8 \\
\hline Supportive care sub-scale score & $\mathrm{N}=450$ & \\
\hline Low & 111 & 24.7 \\
\hline Medium & 196 & 43.6 \\
\hline High & 143 & 31.8 \\
\hline PCMC = Person-centred maternity care & & \\
\hline aPercentages may not add up to 100 due to rounding & \\
\hline
\end{tabular}


Table 4

Descriptive statistics of person-centred maternity care $(\mathrm{N}=450)$

\begin{tabular}{|lccccccc|}
\hline PCMC domain & Minimum & Maximum & Mean & $\begin{array}{l}\text { Std. } \\
\text { Deviation }\end{array}$ & & \multicolumn{2}{l|}{ Percentiles } \\
\cline { 5 - 8 } & & & & & 25th & 75th \\
\hline Total PCMC score & 15 & 83 & 55.13 & 17.83 & 54.00 & 67.00 \\
\hline Dignity and respect & 2 & 18 & 10.27 & 3.43 & 9.00 & 12.00 \\
$\begin{array}{l}\text { Communication and } \\
\text { Autonomy }\end{array}$ & 3 & 27 & 18.14 & 6.38 & 15.00 & 23.00 \\
\hline $\begin{array}{l}\text { Supportive care } \\
\text { PCMC = person-centred maternity care }\end{array}$ & 6 & 42 & 26.67 & 8.94 & & 26.00 & 32.00 \\
\hline
\end{tabular}

\section{Bivariate analysis}

Table 5 shows mean score differences in PCMC disaggregated by predictors. Women who married at age $\geq 30$ years had significantly lower PCMC score than those who married before 30 years $(\rho=0.000)$. Selfemployed women $(\rho=0.000)$ and women married to unemployed partners $(\rho=0.027)$ had significantly the least PCMC scores among occupational categories. Women with high participation in household decision-making had significantly lower PCMC score than those with low participation $(\rho=0.000)$. Women who had experienced domestic violence had significantly lower PCMC score than those with no experience $(\rho=0.001)$. Women who were delivered in health centres had higher PCMC scores than those delivered in public and private hospitals $(\rho=0.000)$. Women who were delivered by doctors had higher PCMC scores than those delivered by other providers $(\rho=0.039)$. Women who had pregnancy complications had higher PCMC scores than those without pregnancy complications $(\rho=0.045)$. Women who started antenatal care during the third trimester had significantly lower PCMC score than other women $(\rho=0.000)$. 
Table 5

Mean score1 (standard deviation) of perceived person-centred maternity care disaggregated by respondents' characteristics

\begin{tabular}{|c|c|c|c|c|}
\hline Parameters & & Mean $^{1}$ & SD & Sig. \\
\hline \multirow[t]{3}{*}{$\mathrm{Age}^{2}$} & $15-19$ years & 54.80 & 18.46 & \\
\hline & $20-29$ years & 55.66 & 17.72 & 0.758 \\
\hline & $30-49$ years & 54.35 & 17.97 & \\
\hline \multirow[t]{2}{*}{ Marital status ${ }^{3}$} & Married & 55.29 & 17.72 & 0.093 \\
\hline & Not married & 43.00 & 23.30 & \\
\hline \multirow[t]{2}{*}{ Religion ${ }^{3}$} & Christianity & 55.21 & 17.82 & 0.264 \\
\hline & Muslim & 43.67 & 18.77 & \\
\hline \multirow[t]{2}{*}{ Ethnicity $^{3}$} & Igbo & 55.16 & 17.90 & 0.717 \\
\hline & Others & 52.50 & 11.22 & \\
\hline \multirow[t]{2}{*}{ Residence $^{3}$} & Urban & 54.70 & 17.56 & 0.612 \\
\hline & Rural & 55.56 & 18.12 & \\
\hline \multirow[t]{3}{*}{ Age at marriage ${ }^{2}$} & $15-19$ years & 50.37 & 20.09 & \\
\hline & $20-29$ years & 57.58 & 16.06 & $0.000 *$ \\
\hline & $30-49$ years & 45.20 & 21.22 & \\
\hline \multirow[t]{4}{*}{ Parity $^{2}$} & 1 & 55.51 & 17.91 & \\
\hline & 2 & 57.00 & 16.77 & 0.171 \\
\hline & 3 & 51.86 & 18.42 & \\
\hline & $\geq 4$ & 55.63 & 18.33 & \\
\hline \multicolumn{5}{|c|}{ Educational level of respondent ${ }^{2}$} \\
\hline & No school/primary & 53.96 & 18.63 & \\
\hline & Secondary/Vocational & 55.55 & 17.86 & 0.809 \\
\hline \multicolumn{5}{|c|}{${ }^{1}$ Reported on scale of $0-90$ with higher scores corresponding to higher PCMC. } \\
\hline \multicolumn{5}{|l|}{${ }^{2}$ ANOVA } \\
\hline \multicolumn{5}{|l|}{3 t-test } \\
\hline *Significant at $\rho \leq 0.05$ & & & & \\
\hline
\end{tabular}




\begin{tabular}{|c|c|c|c|c|}
\hline \multicolumn{2}{|l|}{ Parameters } & \multirow{2}{*}{$\begin{array}{l}\text { Mean }^{1} \\
54.79\end{array}$} & \multirow{2}{*}{$\begin{array}{l}\text { SD } \\
17.52\end{array}$} & \multirow[t]{2}{*}{ Sig. } \\
\hline & Tertiary & & & \\
\hline \multirow[t]{3}{*}{ Literacy rate writing $^{2}$} & No, cannot write & 56.73 & 16.66 & \\
\hline & Yes, with some difficulty & 55.78 & 17.43 & 0.547 \\
\hline & Yes, very well & 54.29 & 18.39 & \\
\hline \multirow[t]{3}{*}{ Literacy rate reading ${ }^{2}$} & No, cannot read & 56.79 & 16.84 & \\
\hline & Yes, with some difficulty & 55.19 & 17.91 & 0.720 \\
\hline & Yes, very well & 54.68 & 18.05 & \\
\hline \multirow[t]{5}{*}{ Wealth index ${ }^{2}$} & Poorest & 57.76 & 18.22 & \\
\hline & Poorer & 53.29 & 20.28 & \\
\hline & Middle & 51.85 & 19.02 & 0.639 \\
\hline & Richer & 56.71 & 16.88 & \\
\hline & Richest & 55.01 & 17.76 & \\
\hline \multirow[t]{5}{*}{ Occupation of respondent ${ }^{2}$} & Unemployed/student & 55.09 & 18.31 & \\
\hline & Self employed & 49.34 & 20.89 & \\
\hline & Salaried worker & 58.05 & 16.14 & $0.000 *$ \\
\hline & Artisan/petty trading & 60.56 & 10.65 & \\
\hline & Farmer & 57.95 & 15.97 & \\
\hline \multirow[t]{2}{*}{ Employment of respondent ${ }^{2}$} & Unemployed & 55.09 & 18.31 & 0.982 \\
\hline & Employed & 55.14 & 17.73 & \\
\hline \multirow[t]{4}{*}{ Partner's occupation ${ }^{2}$} & Unemployed/student & 51.00 & 19.75 & \\
\hline & Self employed & 52.38 & 20.04 & \\
\hline & Salaried worker & 56.38 & 17.21 & $0.027 *$ \\
\hline & Artisan/petty trading & 58.91 & 12.18 & \\
\hline \multicolumn{5}{|c|}{${ }^{1}$ Reported on scale of $0-90$ with higher scores corresponding to higher PCMC. } \\
\hline \multicolumn{5}{|l|}{${ }^{2}$ ANOVA } \\
\hline \multicolumn{5}{|l|}{3 t-test } \\
\hline *Significant at $\rho \leq 0.05$ & & & & \\
\hline
\end{tabular}




\begin{tabular}{|c|c|c|c|c|}
\hline \multicolumn{2}{|l|}{ Parameters } & \multirow{2}{*}{$\begin{array}{l}\text { Mean' } \\
57.08\end{array}$} & \multirow{2}{*}{$\begin{array}{l}\text { SD } \\
17.55\end{array}$} & \multirow[t]{2}{*}{ Sig. } \\
\hline & Farmer & & & \\
\hline \multicolumn{5}{|l|}{ Partner's educational level ${ }^{2}$} \\
\hline & No school/primary & 52.99 & 19.72 & 0.426 \\
\hline & Secondary/Vocational & 55.96 & 17.11 & \\
\hline & Tertiary & 55.06 & 17.79 & \\
\hline \multirow[t]{2}{*}{ Health insurance 3} & No & 55.18 & 17.804 & 0.828 \\
\hline & Yes & 54.53 & 18.299 & \\
\hline \multirow[t]{2}{*}{ Participation in decision making ${ }^{3}$} & Low participation & 61.60 & 10.31 & $0.000^{*}$ \\
\hline & High participation & 46.44 & 21.72 & \\
\hline \multirow[t]{2}{*}{ Domestic violence attitude ${ }^{3}$} & DV tolerance & 57.31 & 14.42 & 0.387 \\
\hline & DV intolerance & 54.89 & 18.16 & \\
\hline \multirow[t]{2}{*}{ Domestic violence experience ${ }^{3}$} & No & 59.39 & 19.38 & $0.001 *$ \\
\hline & Yes & 53.32 & 12.56 & \\
\hline \multirow[t]{3}{*}{ Delivery facility Type ${ }^{2}$} & Public hospital & 49.48 & 19.49 & \\
\hline & Public health centre & 62.08 & 9.90 & $0.000 *$ \\
\hline & Private/mission & 55.14 & 19.15 & \\
\hline \multirow[t]{4}{*}{ Provider type ${ }^{2}$} & Nurse/midwife & 56.30 & 17.68 & \\
\hline & Doctor & 56.73 & 16.38 & $0.039 *$ \\
\hline & Community health worker & 54.60 & 17.35 & \\
\hline & Non-skilled attendant & 48.35 & 20.99 & \\
\hline \multirow[t]{2}{*}{ Pregnancy complication ${ }^{3}$} & No & 54.62 & 18.20 & $0.045^{\star}$ \\
\hline & Yes & 60.76 & 11.67 & \\
\hline Previous facility delivery ${ }^{3}$ & No & 55.67 & 17.94 & 0.614 \\
\hline \multicolumn{5}{|c|}{${ }^{1}$ Reported on scale of $0-90$ with higher scores corresponding to higher PCMC. } \\
\hline \multicolumn{5}{|l|}{${ }^{2}$ ANOVA } \\
\hline \multicolumn{5}{|l|}{$3_{\text {t-test }}$} \\
\hline *Significant at $\rho \leq 0.05$ & & & & \\
\hline
\end{tabular}




\begin{tabular}{|c|c|c|c|c|}
\hline \multicolumn{2}{|l|}{ Parameters } & \multirow{2}{*}{$\begin{array}{l}\text { Mean }^{1} \\
54.79\end{array}$} & \multirow{2}{*}{$\begin{array}{l}\text { SD } \\
17.78\end{array}$} & \multirow[t]{2}{*}{ Sig. } \\
\hline & Yes & & & \\
\hline \multirow[t]{4}{*}{ Trimester of first $\mathrm{ANC}^{2}$} & No ANC & 45.60 & 19.89 & \\
\hline & First trimester & 55.45 & 17.26 & \\
\hline & Second trimester & 57.04 & 16.79 & $0.000 *$ \\
\hline & Third trimester & 41.94 & 22.16 & \\
\hline \multirow[t]{4}{*}{ Number of ANC visits ${ }^{2}$} & No ANC & 53.13 & 18.35 & \\
\hline & $<4$ & 51.00 & 19.11 & \\
\hline & 4 or 5 & 56.56 & 16.52 & 0.367 \\
\hline & $\geq 6$ & 54.88 & 18.43 & \\
\hline \multirow[t]{5}{*}{ Postpartum length ${ }^{2}$} & 1 week & 58.58 & 13.54 & \\
\hline & 2 weeks & 59.67 & 18.77 & 0.338 \\
\hline & 3 weeks & 50.00 & 15.28 & \\
\hline & 4 weeks & 58.88 & 15.50 & \\
\hline & 5-9 weeks & 54.39 & 18.38 & \\
\hline \multicolumn{5}{|c|}{${ }^{1}$ Reported on scale of $0-90$ with higher scores corresponding to higher PCMC. } \\
\hline \multicolumn{5}{|l|}{${ }^{2}$ ANOVA } \\
\hline \multicolumn{5}{|l|}{$3_{\text {t-test }}$} \\
\hline *Significant at $\rho \leq 0.05$ & & & & \\
\hline
\end{tabular}

\section{Predictors of person-centred maternity care}

Table 6 shows the parameters that predicted perceived PCMC among women in this study. Marrying at age $20-29$ years $(\beta=5.83, \rho=0.002)$, self-employed women $(\beta=-10.23, \rho=0.004)$, starting antenatal care in the third trimester $(\beta=-7.93, \rho=0.008)$, high participation in household decisions $(\beta=-4.98, \rho=$ $0.011)$, domestic violence experience $(\beta=5.41, \rho=0.002)$, delivery at health centre $(\beta=7.09, \rho=0.000)$, delivery at private/mission hospital $(\beta=17.47, \rho=0.000)$, delivery by non-skilled attendant $(\beta=-9.61, \rho=$ $0.001)$; delivery by community health worker $(\beta=-7.65, \rho=0.001)$, and experience of pregnancy complication $(\beta=6.20, \rho=0.028)$ predicted PCMC. 
Table 6

Predictors of PCMC among childbearing women in Enugu, Nigeria, 2019

\begin{tabular}{|c|c|c|c|c|c|}
\hline \multirow[t]{2}{*}{ Predictor variables } & & \multirow[t]{2}{*}{ B } & \multicolumn{2}{|c|}{$\begin{array}{l}\text { 95\% Confidence } \\
\text { Interval }\end{array}$} & \multirow[b]{2}{*}{ Sig. } \\
\hline & & & Lower & Upper & \\
\hline & (Intercept) & 51.43 & 43.85 & 59.02 & 0.000 \\
\hline \multirow[t]{3}{*}{ Age at marriage } & $15-19$ years & & & & \\
\hline & $20-29$ years & 5.83 & 2.12 & 9.53 & $0.002^{*}$ \\
\hline & $30-49$ years & -3.71 & -9.85 & 2.44 & 0.237 \\
\hline \multirow[t]{5}{*}{ Occupation of respondent } & Farmer & $0^{\mathrm{a}}$ & & & \\
\hline & Unemployed/students & -5.72 & -12.88 & 1.44 & 0.118 \\
\hline & Self-employed & -10.23 & -17.17 & -3.29 & $0.004 *$ \\
\hline & Salaried (govt.) & -2.78 & -10.40 & 4.84 & 0.475 \\
\hline & Artisan/petty trader & -3.30 & -9.85 & 3.24 & 0.322 \\
\hline \multirow[t]{5}{*}{ Partner occupation } & Farmer & $0^{\mathrm{a}}$ & & & \\
\hline & Unemployed/students & 5.44 & -4.64 & 15.51 & 0.290 \\
\hline & Self-employed & 5.00 & -2.42 & 12.42 & 0.186 \\
\hline & Salaried (govt.) & 6.04 & -1.81 & 13.89 & 0.131 \\
\hline & Artisan/petty trader & 4.12 & -2.76 & 11.01 & 0.241 \\
\hline \multirow[t]{4}{*}{ Initiation of ANC } & First trimester & $0^{a}$ & & & \\
\hline & Second trimester & 0.62 & -2.41 & 3.64 & 0.690 \\
\hline & Third trimester & -7.93 & -13.80 & -2.07 & $0.008^{*}$ \\
\hline & No ANC & -9.36 & -23.37 & 4.65 & 0.190 \\
\hline \multirow[t]{2}{*}{ Participation in decisions } & Low participation & $0^{a}$ & & & \\
\hline & High participation & -4.98 & -8.82 & -1.15 & $0.011 *$ \\
\hline \multirow[t]{2}{*}{ Domestic violence (DV) } & No DV experience & $0^{\mathrm{a}}$ & & & \\
\hline & DV experience & 5.41 & 1.98 & 8.83 & $0.002^{*}$ \\
\hline \multirow[t]{2}{*}{ Delivery facility type } & Public hospital & $0^{\mathrm{a}}$ & & & \\
\hline & Public health centre & 7.09 & 3.39 & 10.79 & $0.000 *$ \\
\hline
\end{tabular}




\begin{tabular}{|c|c|c|c|c|c|}
\hline \multicolumn{2}{|l|}{ Predictor variables } & \multirow[t]{2}{*}{ B } & \multicolumn{3}{|c|}{$\begin{array}{l}\text { 95\% Confidence } \\
\text { Interval }\end{array}$} \\
\hline & & & Lower & Upper & Sig. \\
\hline & $\begin{array}{l}\text { Private/mission } \\
\text { hospital }\end{array}$ & 17.47 & 12.59 & 22.35 & $0.000 *$ \\
\hline \multirow[t]{4}{*}{ Provider type } & Nurse/Midwife & $0^{\mathrm{a}}$ & & & \\
\hline & Non-skilled attendant & -9.61 & -15.44 & -3.78 & $0.001 *$ \\
\hline & $\begin{array}{l}\text { Community health } \\
\text { worker }\end{array}$ & -7.65 & -12.33 & -2.97 & $0.001 *$ \\
\hline & Doctor & 0.04 & -4.64 & 4.72 & 0.986 \\
\hline \multirow{3}{*}{$\begin{array}{l}\text { Pregnancy complication } \\
\text { (PC) }\end{array}$} & No PC experience & $0^{\mathrm{a}}$ & & & \\
\hline & PC experience & 6.20 & 0.66 & 11.74 & $0.028 *$ \\
\hline & (Scale) & $229.57^{b}$ & 201.45 & 261.62 & \\
\hline \multicolumn{6}{|c|}{ a. Set to zero because this parameter is reference. } \\
\hline \multicolumn{6}{|c|}{ b. Maximum likelihood estimate. } \\
\hline *Significance at $\rho \leq 0.05$ & & & & & \\
\hline
\end{tabular}

\section{Discussion}

The study found that women had medium scores on full PCMC scale and sub-scales. The least score was communication and autonomy sub-scale, while highest score was respect and dignity dimension. Our findings are comparable to evidence from previous studies $[1,6,10,11]$. Although, most women in this study were treated with respect, as much as $20 \%$ were verbally abused, which is slightly higher than findings of $11-18 \%$ in prior studies $[6,10,11]$, but consistent with high incidence of disrespect and abuse in other studies [12-19]. Similarly, our finding that $7 \%$ of women were physically abused was higher than results elsewhere $[6,10,11,15]$, but lower than findings reported in previous studies [12,16-18]. The sharp decrease in physical abuse might reflect underreporting as this behaviour may be accepted as normal and not considered as abuse or disrespect by some women in Nigeria [16], or due to a rising awareness of litigation among care providers [15]. Lower score on communication and autonomy is accounted for mainly by a quarter of women who received limited consented care, explanation of procedure and medicines; and another third who were least involved in their care, not called by name and received inappropriate introduction from providers. Conversely, previous studies found higher prevalence of unconsented care, limited explanation of medicine and procedures, not calling of women by name and inappropriate introduction by providers $[6,10,11]$. Consistent with other studies $[6,10,11]$, women were greatly concerned about lack of labour support, restrictive childbirth companionship and inadequate 
staffing. While many areas of strength exist across the three domains of PCMC, meaningful changes to improve PCMC would involve reducing verbal and physical abuse, improving communication with women, and addressing gaps in facility-level drivers of PCMC.

This study revealed that marriage at 20-29 years had a significant positive relationship with women's perception of person-centred maternity care, which is consistent with a higher incident of disrespect and abuse among women aged 20-34 years [22]. The observed relation between age at marriage and PCMC might not simply reflect age, but also economic and educational empowerment given that nearly $60 \%$ of women in our sample belong to rich quintiles and majority of women have a minimum of secondary education. In Nigeria, women with no education marry six years earlier than women with secondary education, whereas women in the lowest wealth quintile marry more than eight years earlier than women in the highest quintile [34]. We argue that women in this study, who marry at age 20-29 years, are better empowered, more likely to live in areas with better quality of care, have higher expectation of care and can recognise low-quality care and advocate for improved care [21].

We found that self-employment had significant, but an inverse relationship with women's perception of person-centred maternity care. An increase in self-employment would result in decrease in PCMC among self-employed women. This finding is comparable with evidence in Kenya which found that employment status predicted women's perception of PCMC [8]. However, while the study in Kenya dichotomized occupation into unemployed and employed, our study used five occupational categories. Two factors could explain our findings. First self-employment could enhance women's participation in household decision-making for their own healthcare [41]. Secondly, self-employment increases women's economic empowerment, which means that women can effectively demand better maternity care [21, 41]. As perception of PCMC varies with socio-economic status $[8,11]$, an increase in women's labour participation that promotes self-employment is needed to improve person-centred maternity care.

High participation in household decision-making was found to have an inverse relationship with women's perception of PCMC in this study. Women with high participation have significantly lower PCMC score than those with low participation in household decisions. Our results contrast findings of a prior study in Kenya which found that PCMC was not significantly related to participation in household decisionmaking [8]. In Nigeria, healthcare decisions for women are mostly made by their husbands/partners without women's involvement [42]. It might be that low women's decision-making autonomy limits women's expectation of quality of maternity care, social power between women and providers, and women's capacity to demand better care in Nigeria. Conversely, women who participate highly in household decisions are better aware of their rights to person-centred care and tend to have increased self-confidence thereby reducing power differential between health providers and women [16].

This study further revealed that the experience of domestic violence was inversely related to women's perception of person-centred maternity care. Women who had no domestic violence experience had significantly higher PCMC score than those who experienced domestic violence. Our findings compare to a similar association of domestic violence experience and PCMC in Kenya [8]. These findings are 
expected because women who experience gender-based violence are disempowered and more vulnerable to dominance by providers who use coercive strategies to control women or punish women for perceived disobedience during childbirth [43]. Women who experience domestic violence are emotionally challenged. Women even when receiving technically sound care but lacking in emotional support perceive it as low-quality care $[23,44]$. In Nigeria, childbearing women patronise providers with sympathy and compassion regardless of their level of competence [16]. Also, domestic violence limits women's decisionmaking power regarding their reproductive health and have been associated with poor maternal health outcomes [45]. For instance, Sipsma et al found that women's experience of physical abuse is associated with inadequate use of antenatal care [46].

Trimester of commencing antenatal care predicted women's perception of PCMC in this study. Women who commenced antenatal care during the third trimester were more likely to have a lower perception of person-centred maternity care than women who started antenatal care in their first trimester. Our findings are inconsistent with a previous Kenyan study showing that the onset of antenatal care is not significantly related to the perception of PCMC [8]. Every pregnant woman in developing countries should seek antenatal care during the first trimester of pregnancy because failure to initiate antenatal care early is a potential risk for complications during pregnancy and childbirth [47]. Tailored group educational activities and peer support motivates behaviour change among pregnant women and increases women's satisfaction with maternity care [47]. In this study, late initiation of antenatal care meant that women are not familiar with the health system and might not have the benefit of psychological support and sharing of experiences which help women feel more empowered as decision makers during childbirth [48].

Moreover, women who were delivered in health centres and private/mission hospitals had higher PCMC scores than those delivered in public hospitals. Similar findings of higher PCMC were also found in health centres and private hospitals in Kenya [8]. Also, mothers who gave birth in primary level facilities also tended to be more satisfied than those who gave birth in hospitals [29]. In Pakistan, the risk of reporting disrespect and abuse was also higher in public than private hospitals [23]. Our finding also compares to other studies that find higher interpersonal quality of maternal healthcare in private than public hospitals $[25,28]$. Conversely, indices of clinical quality of maternal health were higher in public hospitals than private hospitals and health centres [25-27]. In this study, higher PCMC scores in health centres and private hospitals may be due to low provider-patient ratio which reduces the strain on provider-patient interaction [8]. Equally, higher PCMC scores in health centres might reflect closer ties between providers and women in closely knitted communities that health centres serve [8] and effect of citizen participation in governance of health centres [49]. In Nigeria, users have better perception of health workers in private facilities because private facilities greatly emphasize interpersonal quality [50].

Type of birth attendant was also found to predict women's perception of PCMC in this study. PCMC was inversely and significantly related to delivery by community health workers and non-skilled attendants, although we expected a direct relationship given that negative attitudes and behaviours are commonly ascribed to trained professionals especially doctors and nurses [51]. Although women who were delivered by doctors received higher PCMC than those delivered by nurses, delivery by doctors was not significantly 
predictive. By contrast, PCMC was directly and significantly related to delivery by doctors in Kenya [8]. Higher perception of PCMC among women delivered by doctors than nurses is consistent with a Nigerian study showing that healthcare users have a better perception of doctors than nurses [50]. It could be that negative attitudes and behaviours are more common among nurses than doctors as hostile and impersonal behaviour from nurses and midwives are common reasons for dissatisfaction with quality of maternal health services in South-east Nigeria [52].

Furthermore, our study revealed that women who had pregnancy complications had higher PCMC scores than those without pregnancy complications; and experience of pregnancy complication significantly predicted perception of PCMC. Comparable results were found in Kenya, where women with severe pregnancy complication reported higher PCMC than other women [8]. By contrast, we expected that women with pregnancy complications will have significantly lower PCMC than those without complications. Our expectation is consistent with findings in previous studies showing that there were higher incidents of disrespect and abuse among women who experience pregnancy complications and longer labour durations requiring instrumental delivery and caesarean birth [19, 22, 30-32]. It could be that survivors of pregnancy complication are more satisfied with their positive pregnancy outcomes and tend to report exaggerated positive patient experiences.

There is little evidence on the determinants of person-centred maternity care using a validated tool [8]. Hence, this study adds to the nascent scholarship on factors influencing disparities in patients' experiences of maternity care in low- and middle-income countries. This notwithstanding, this study has some potential limitations. First, the study was limited to two health districts in one Nigerian state. The health facilities from which women were sampled are not representative of the entire state as nonprobability sampling approaches were used. Thus, respondents cannot be generalisable to all women. Second, recall bias is common in retrospective data collection, though respondents in this study seemed to recall experiences of their pregnancy and childbirth vividly. Notwithstanding that women could recall childbirth experiences accurately within twenty years [53], we adopted 9 weeks post-partum used in a previous study [1]. Third, there may also have been sampling bias as only women who gave birth to live babies and attended immunization clinics were included. The study, therefore, potentially excluded women with stillbirths and neonatal deaths who may have had more negative childbirth experiences.

\section{Conclusion}

The purpose of this study was to assess predictors of PCMC in Enugu State, South-east Nigeria. Evidence from this study highlight the role five patient characteristics (age at marriage, self-employed women, high participation in household decisions, domestic violence experience and initiation of antenatal care in the third trimester); two facility characteristics (facility type and provider type); and service type (pregnancy complication) as predictors of person-centred maternity care. This information should inform the design of interventions to promote positive childbirth experiences and can be used to evaluate changes in the quality of maternity care. 


\section{Abbreviations}

ANOVA

Analysis of variance; PCMC:Person-centred maternity care; WCBA:Women of childbearing age.

\section{Declarations}

\section{Ethical approval and consent to participate}

The study was approved by Health Research Ethics Committee of University of Nigeria Teaching Hospital, Enugu, Nigeria. Written informed consent was obtained from all participants for both participation and audio-recording of interviews. Written informed consent for participation in the study was obtained, where participants are children (under 16 years old) from their parent or guardian.

\section{Consent for publication}

Not applicable.

\section{Availability of data and materials}

The datasets generated and/or analysed during the current study are not publicly available but are available from the corresponding author on reasonable request.

\section{Competing interests}

The authors report no conflict of interest.

\section{Funding}

This work received no external funding.

\section{Authors' contributions}

DCO and CN contributed to conceptualisation of the study. DCO and CN conducted the field work. DCO analysed the data and drafted the manuscript. DCO and CN contributed to the intellectual content of the article. DCO and CN finalised the article. All authors read and approved the final manuscript.

\section{Acknowledgements}


We are grateful to Metrics for Management for allowing us to use the Nigeria equity tool and giving us the syntax for analysing the data.

\section{References}

1. Afulani PA, Diamond-Smith N, Golub G, Sudhinaraset M. Development of a tool to measure personcentered maternity care in developing settings: validation in a rural and urban Kenyan population. Reprod Health. 2017;14(1):118.

2. Miller S, Abalos E, Chamillard M, Ciapponi A, Colaci D, Comandé $D$, et al. Beyond too little, too late and too much, too soon: a pathway towards evidence-based, respectful maternity care worldwide. Lancet. 2016;388(10056):2176-92.

3. Downe S, Lawrie TA, Finlayson K, Oladapo OT. Effectiveness of respectful care policies for women using routine intrapartum services: a systematic review. Reprod Health. 2018;15(1):23.

4. Shakibazadeh E, Namadian M, Bohren MA, Vogel JP, Rashidian A, Nogueira Pileggi V, et al. Respectful care during childbirth in health facilities globally: a qualitative evidence synthesis. Brit $J$ Obstet Gynaecol. 2018;125:932-42.

5. Larson E, Sharma J, Bohren MA, Tunçalp Ö. When the patient is the expert: measuring patient experience and satisfaction with care. Bull World Health Organ. 2019;97:563-9.

6. Afulani PA, Diamond-Smith N, Phillips B, Singhal S, Sudhinaraset M. Validation of the personcentered maternity care scale in India. Reprod Health. 2018;15(1):147.

7. WHO. WHO recommendations: intrapartum care for a positive childbirth experience. Geneva: World Health Organisation; 2018.

8. Afulani PA, Sayi TS, Montagu D. Predictors of person-centered maternity care: the role of socioeconomic status, empowerment, and facility type. BMC Health Serv Res. 2018;18(1):360.

9. Afulani P, Moyer C. Explaining disparities in use of skilled birth attendants in developing countries: a conceptual framework. PLoS One. 2016;11:e0154110.

10. Sudhinaraset M, Giessler K, Golub G, Afulani P. Providers and women's perspectives on personcentered maternity care: a mixed methods study in Kenya. Int J Equity Health. 2019;18:83.

11. Afulani P, Phillips B, Aborigo RA, Moyer CA. Person-centred maternity care in low-income and middleincome countries: analysis of data from Kenya, Ghana, and India. Lancet. 2019;7:e96-109.

12. Okafor II, Ugwu EO, Obi SN. Disrespect and abuse during facility-based childbirth in a low-income country. Int J Gynaecol Obstet. 2015;28(2):110-3.

13. Idris SH, Sambo MN, Ibrahim MS. Barriers to utilisation of maternal health services in a semi-urban community in northern Nigeria: the clients' perspective. Niger Med J. 2013;54(1):27-32.

14. Moore BM, Alex-Hart BA, George IO. Utilisation of health care services by pregnant mothers during delivery: a community-based study in Nigeria. East Afr J Public Health. 2011;8(1):49-51.

15. ljadunola MY, Olotu EA, Oyedun OO, Eferakeya SO, llesanmi Fl, Fagbemi AT, et al. Lifting the veil on disrespect and abuse in facility-based child birth care: findings from South West Nigeria. BMC 
Pregnancy Childbirth. 2019;19:39.

16. Ishola F, Owolabi O, Filippi V. Disrespect and abuse of women during childbirth in Nigeria: A systematic review. PLoS One. 2017;12(3):e0174084.

17. Bohren MA, Mehrtash H, Fawole B, Maung TM, Balde MD, Maya E, et al. How women are treated during facility-based childbirth in four countries: a cross-sectional study with labour observations and community-based surveys. Lancet. 2019;394:1750-63.

18. Bobo FT, Kasaye HK, Etana B, Woldie M, Feyissa TR. Disrespect and abuse during childbirth in Western Ethiopia: Should women continue to tolerate? PLoS One. 2019;14(6):e0217216.

19. Wassihun B, Zeleke S. Compassionate and respectful maternity care during facility based child birth and women's intent to use maternity service in Bahir Dar, Ethiopia. BMC Pregnancy Childbirth. 2018;18(1):294.

20. Bleich SN, Ozaltin E, Murray CK. How does satisfaction with the healthcare system relate to patient experience? Bull World Health Organ. 2009;87(4):271-8.

21. Afulani PA. Conceptualizing pathways linking women's empowerment and prematurity in developing countries. BMC Pregnancy Childbirth. 2017;17:338.

22. Gebremichael MW, Worku A, Medhanyie AA, Berhane Y. Mothers' experience of disrespect and abuse during maternity care in northern Ethiopia. Glob Health Action. 2018;11(sup3):1465215.

23. Azhar Z, Oyebode O, Masud H. Disrespect and abuse during childbirth in district Gujrat, Pakistan: A quest for respectful maternity care. PLoS One. 2018;13(7):e0200318.

24. WHO. Standards for improving quality of maternal and newborn care in health facilities. Geneva: World Health Organisation; 2016.

25. Diamond-Smith N, Sudhinaraset M, Montagu D. Clinical and perceived quality of care for maternal, neonatal and antenatal care in Kenya and Nambia: the service provision assessment. Reprod Health. 2016;13:92.

26. Boller C, Wyss K, Mtasiwa D, Tanner M. Quality and comparison of antenatal care in public and private providers in the United Republic of Tanzania. Bull World Health Organ. 2003;81:116-22.

27. Hutchinson PL, Do M, Agha S. Measuring client satisfaction and quality of family planning services: a comparative analysis of public and private health facilities in Tanzania, Kenya and Ghana. BMC Health Serv Res. 2011;11:203.

28. Afulani PA. Rural/urban and socio-economic differentials in quality of antenatal care in Ghana. PLoS One. 2015;10:e117996.

29. Mocumbi S, Högberg U, Lampa E, Sacoor C, Valá A, Bergström A, et al. Mothers' satisfaction with care during facility-based childbirth: a cross-sectional survey in southern Mozambique. BMC Pregnancy Childbirth. 2019;19:303.

30. Colley S, Kao C, Gau M, Cheng S. Women's perception of support and control in The Gambia, a quantitative study on dignified facility-based intrapartum care. BMC Pregnancy Childbirth. 2018;18:413. 
31. Galle A, Manaharlal H, Cumbane E, Picardo J, Griffin S, Osman N, et al. Disrespect and abuse during facility-based childbirth in southern Mozambique: a cross-sectional study. BMC Pregnancy Childbirth. 2019;19:369.

32. Banks KP, Karim AM, Ratcliffe HL, Betemariam W, Langer A. Jeopardizing quality at the frontline of healthcare: prevalence and risk factors for disrespect and abuse during facility-based childbirth in Ethiopia. Health Policy Plann. 2018;33:317-27.

33. Ogbuabor DC, Onwujekwe OE. Implementation of free maternal and child healthcare policies: assessment of influence of context and institutional capacity of health facilities in South-east Nigeria. Glob Health Action. 2018;11:1535031.

34. NPC [Nigeria] and ICF. Nigeria demographic and health survey 2018. Nigeria: National Population Commission and ICF;:: Abuja; 2019.

35. Ezugwu EC, Agu PC, Nwoke MO, Ezugwu FO. Reducing maternal deaths in a low resource setting in Nigeria. NigerJ Clin Pract. 2014;17(1):62-6.

36. Pituch KA, Stevens JP. Applied multivariate statistics for the social sciences. 6th ed. New York: Routledge/Taylor \& Francis Group; 2016.

37. Pett MA. Nonparametric Statistics for Health Care Research: Statistics for Small Samples and Unusual Distributions (NULL). 2nd ed. California: Thousand Oaks, Sage Publications; 2015.

38. Sudhinaraset M, Landrian A, Afulani PA, Diamond-Smith N, Golub G. Association between personcentered maternity care and newborn complications in Kenya. Int J Gynaecol Obstet. 2019;1:1-8.

39. Metrisc for Management. Nigeria equity tool: Metric for Measurement; 2015 [Available from: https://www.equitytool.org/nigeria/.

40. Musonera A, Heshmati A. Measuring women's empowerment in Rwanda. Institute for the Study of Labour (IZA); 2016. Contract No.: 10131.

41. Asaolu IO, Alaofe H, Gunn JKL, Adu AK, Monroy AJ, Ehiri JE, et al. Measuring women's empowerment in sub-Saharan Africa: exploratory and confirmatory factor analysis of the demographic and health surveys. Front Psychol. 2018;9:994.

42. Osamor P, Grady C. Factors associated with women's healthcare decision-making autonomy: empirical evidence from Nigeria. J Biosoc Sci. 2017;50(1):70-85.

43. Jewkes R, Penn-Kekana L. Mistreatment of women in childbirth: time for action on this important dimension of violence against women. PLoS Med. 2015;12(6):e1001849.

44. Bohren MA, Vogel JP, Hunter EC, Lutsiv O, Makh SK, Souza JP, et al. The Mistreatment of Women during Childbirth in Health Facilities Globally: A Mixed-Methods Systematic Review. PLoS Med. 2015;12(6):e1001847.

45. Benebo FO, Schumann B, Vaezghasemi M. Intimate partner violence against women in Nigeria: a multilevel study investigating the effect of women's status and community norms. BMC Women Health. 2018;18:136. 
46. Sipsma H, Ofori-Atta A, Canavan M, Udry C, Bradley E. Empowerment and use of antenatal care among women in Ghana: a cross-sectional study. BMC Pregnancy Childbirth. 2014;14:364.

47. Manyeh AK, Amu A, Williams J, Gyapong M. Factors associated with the timing of antenatal clinic attendance among first-time mothers in rural southern Ghana. BMC Pregnancy Childbirth. 2020;20:47.

48. Rubashkin N, Warnock R, Diamond-Smith N. A systematic review of person-centered care interventions to improve quality of facility-based delivery. Reprod Health. 2018;15(1):169.

49. Ogbuabor DC, Onwujekwe OE. The community is just a small circle: citizen participation in the free maternal and child healthcare programme of Enugu State, Nigeria. Glob Health Action. 2018;11:1.

50. Odusanya OO, Akinyinka MR, Oluwole EO, Odugbemi BA, Bakare OQ, Adeniran A. How does the public perceive healthcare workers in Lagos? A comparison of health workers in public and private health facilities. Niger Postgrad Med J. 2018;25(3):177-85.

51. Mannava P, Durrant K, Fisher J, Chersich M, Luchters S. Attitudes and behaviours of maternal health care providers in interactions with clients: a systematic review. Global Health. 2015;11:36.

52. Uzochukwu BS, Onwujekwe OE, Akpala CO. Community satisfaction with the quality of maternal and child health services in southeast Nigeria. East Afr Med J. 2004;81(6):293-9.

53. Simkin P. Just another day in woman's life? Part II: Nature and consistency of women's long-term memories of their first birth experiences. Birth. 1992;19:64-81.

\section{Supplementary Files}

This is a list of supplementary files associated with this preprint. Click to download.

- STROBEchecklistcrosssectional.docx 